\title{
ESTRUCTURA Y DIVERSIDAD GENÉTICA EN POBLACIONES DE Pinus engelmannii Carr. EN CHIHUAHUA, MÉXICO
}

\section{GENETIC STRUCTURE AND DIVERSITY OF Pinus engelmannii Carr. POPULATIONS IN CHIHUAHUA, MEXICO}

\section{Carlos R. Morales-Nieto', Minerva Siqueiros-Candia', Alan Álvarez-Holguín², Raúl Corrales-Lerma ${ }^{1}$, Manuel Alarcón-Bustamante ${ }^{2}$ y Martín Martínez-Salvador ${ }^{1}$ *}

\author{
'Universidad Autónoma de Chihuahua, Facultad de Zootecnia y Ecología, Chihuahua, Chihuahua, México. ${ }^{2}$ Instituto Nacional de Investigaciones \\ Forestales, Agrícolas y Pecuarias, Campo Experimental La Campana, Aldama, Chihuahua, México. \\ *Autor de correspondencia (msalvador@uach.mx)
}

\section{RESUMEN}

La evaluación de la estructura y diversidad genética de especies forestales contribuye con la formulación de estrategias para preservar su riqueza genética; a su vez, la riqueza genética contribuye significativamente en la adaptación de las especies ante factores o ambientes nuevos, como aquellos derivados del cambio climático. El objetivo del presente estudio fue evaluar la estructura y diversidad genética de tres poblaciones de Pinus engelmannii Carr. en el estado de Chihuahua, México. Las poblaciones se ubican en los municipios de Madera, Bocoyna y Balleza, en el norte, centro y sur del estado, respectivamente. Se analizaron 30 árboles de las tres poblaciones mediante marcadores moleculares AFLP (polimorfismos en la longitud de fragmentos amplificados). Los resultados revelaron que $P$. engelmannii presenta un nivel de diversidad alto (índice de Shannon $I=0.380$, heterocigosidad esperada $\left.H_{e}=0.249\right)$. La población del centro mostró diferencias $(P \leq 0.05)$ en el nivel de diversidad $\left(I=0.364\right.$ y $\left.H_{e}=0.237\right)$ en comparación de las poblaciones norte y sur. Por su parte, las poblaciones del norte y centro no presentaron diferenciación genética significativa $(P=0.28)$, mientras que la población del sur fue diferente con respecto a las poblaciones del centro $(P<0.045)$ y norte $(P<0.001)$. Los sistemas montañosos y cañones del sur del estado parecen actuar como una barrera geográfica que disminuye el flujo genético de las poblaciones del centro y norte con su contraparte del sur, con valores de 5.74 y $3.38 \mathrm{~N}_{\mathrm{m}}$, respectivamente. La estructura genética encontrada en las poblaciones de $P$. engelmannii sugiere que los programas de reforestación en la región sur del estado de Chihuahua deben llevarse a cabo con materiales locales, mientras que la reforestación para el centro y norte puede realizarse con materiales de ambas regiones, a fin de preservar la estructura y diversidad genética de esta especie.

Palabras clave: Pinus engelmannii, barreras geográficas, flujo genético, marcadores AFLP, relaciones genéticas.

\section{SUMMARY}

The assessment of the genetic structure and diversity of forest species contributes to the formulation of strategies that preserve their genetic richness. In turn, genetic richness significantly contributes to the adaptation of species to new factors or environments derived from climate change. The objective of this study was to assess the structure and genetic diversity of three Pinus engelmannii populations in the state of Chihuahua, Mexico. The populations are located in the municipalities of Madera, Bocoyna and Balleza at the North, Center and South of the state, respectively. Thirty trees from the three populations were analyzed using AFLP (amplified fragment length polymorphism) molecular markers. Results revealed that $P$. engelmannii presents a high level of diversity (Shannon index $I=0.380$, expected heterozygosity $H_{e}=0.249$ ). The population from the center showed differences $(\mathrm{P} \leq 0.05)$ in the level of diversity $\left(I=0.364\right.$ and $\left.H_{e}=0.237\right)$ compared to populations from the North and South. Meanwhile, the Northern and Central populations did not show significant genetic differentiation $(P=0.28)$, while the Southern population was different to the Central $(P<0.045)$ and Northern populations $(P<0.001)$. The mountainous systems and canyons in the South of the state seem to act as a geographic barrier that decreases the genetic flow of the Central and Northern populations with their Sothern counterpart with values of 5.74 and $3.38 \mathrm{~N}_{\mathrm{m}}$, respectively. The genetic structure found in the $P$. engelmannii populations suggests that reforestation programs in the Southern region of the state of Chihuahua should be carried out using local materials. In contrast, reforestation at the Central and Northern areas can be carried out with materials from both regions, in order to preserve the structure and genetic diversity of this species.

Index words: Pinus engelmannii, AFLP markers, genetic flow, genetic relationships, geographic barriers.

\section{INTRODUCCIÓN}

La conservación de recursos genéticos de especies forestales es fundamental para mantener la estabilidad de los bosques (FAO et al., 2007; St.Clair y Howe, 2011), la cual contribuye a la adaptación de estas especies ante la ocurrencia de diferentes factores como la infestación de plagas, los regímenes de incendios o el cambio climático (Kim et al., 2011; Vangestel et al., 2016). Adicionalmente, el conocer la estructura y diversidad genética de especies forestales es importante para la planeación y operación de programas de reforestación, ya que permite diseñar estrategias para el manejo y conservación de recursos genéticos (Bilgen y Kaya, 2017; Pazouki et al., 2016). Por otra parte, conocer la estructura y diversidad genética de especies forestales de importancia económica permite realizar programas de selección para incrementar la productividad y la probabilidad de éxito de las reforestaciones (Ivetić et al., 2016).

Pinus engelmannii Carr. es económicamente importante 
en el norte de México debido a sus propiedades maderables y a su amplia distribución (Ávila-Flores et al., 2016). Esta especie se distribuye en 2,450,000 ha, aproximadamente (CONAFOR, 2009) y se encuentra principalmente en los estados de Chihuahua, Durango, Sinaloa, Sonora y Zacateca (Sánchez-González, 2008). Por su distribución y potencial maderable, $P$. engelmannii es ampliamente utilizada en programas de reforestación (Prieto et al., 2004).

La estructura y diversidad genética se ha evaluado previamente en especies de pinos en el norte de México. Hernández-Velasco et al. (2017) encontraron diferencias genéticas entre poblaciones de $P$. cembroides Zucc. ssp. cembroides, P. discolor D. K. Bailey \& Hawksw, P. durangensis Martínez y P. teocote Schltdl. \& Cham; por su parte, Quiñones-Pérez et al. (2014) y Friedrich et al. (2018) reportaron amplia diversidad genética entre poblaciones de P. arizonica (Engelm.) Shaw y Picea chihuahuana Martínez. De igual manera, Ortiz-Olivas et al. (2017) evaluaron la diversidad genética dentro de rodales semilleros de $P$. engelmannii y encontraron diferencias genéticas entre las localidades evaluadas en el estado de Durango. La diversidad y estructura genética también se han evaluado a nivel de huertos semilleros en Pinus patula Schiede ex Schltdl. et Cham (Méndez-Neri et al., 2020), donde se ha encontrado que la mayor diversidad genética se encuentra dentro de los huertos.

En el estado de Chihuahua P. engelmannii se distribuye a lo largo de la Sierra Madre Occidental, con secciones discontinuas generadas por las barrancas en diferentes regiones del estado; estas barreras geográficas y la amplia distribución de la especie podrían generar poblaciones con diferencias en diversidad genética a lo largo del estado. Bajo este marco, el objetivo del presente estudio fue conocer la estructura y diferenciación genética de tres poblaciones de Pinus engelmannii en el estado de Chihuahua.

\section{MATERIALES Y MÉTODOS}

\section{Área de estudio y método de muestreo}

La toma de muestras se realizó en tres sitios del estado de Chihuahua (Figura 1). Los sitios de muestreo se ubicaron en los bosques templados de las zonas norte, centro y sur del estado, en los municipios de Madera, Bocoyna y Balleza, respectivamente (Figura 1; Cuadro 1). En cada población se recolectaron hojas (acículas) de 10 individuos seleccionados al azar en el centro de diferentes rodales de masas puras de la especie. Se consideró una distancia promedio de $1 \mathrm{~km}$ entre cada árbol. Las muestras de acículas se colocaron en bolsas de plástico y se transportaron en hieleras al laboratorio de Genética Molecular del Centro de Investigación y de Estudios
Avanzados del Instituto Politécnico Nacional (CINVESTAV), Unidad Irapuato, Guanajuato.

El tamaño de muestra se basa en los criterios propuestos por Rodríguez-Peña et al. (2018), quienes sugirieron que la técnica de AFLP es muy útil para realizar estudios de estructuras genéticas, pues es posible economizar utilizando muestras relativamente pequeñas; así mismo, Nei (1978) indicó que es posible estimar heterocigosidad promedio y distancia genética con cualquier tamaño de muestra, siempre y cuando se considere un alto número de loci.

\section{Extracción y análisis de ADN}

Para la obtención de ADN se utilizaron de dos a tres acículas por árbol; se utilizó el kit Qiagen DNeasy® 96 Plant Kit (QIAGEN Inc, Alemania). El material se molió en un mortero, el cual se desinfectó con etanol 70 \% entre cada muestra. El material molido se colocó en tubos de microcentrífuga de $2 \mathrm{~mL}$, junto con una esfera de metal de $5 \mathrm{~mm}$ (Qiagen Stainless Steel Beads). Posteriormente, las muestras fueron liofilizadas y re-molidas en un disruptor/ homogeneizador de muestras TissueLyser II (Quiagen ${ }^{\circledR}$ Inc., Haan, Alemania) con dos ciclos de $30 \mathrm{~Hz}$ por $30 \mathrm{~s}$. Las muestras molidas y liofilizadas fueron utilizadas para la extracción del ADN. Una vez realizada la extracción, el total de ADN genómico se cuantificó en un espectrofotómetro

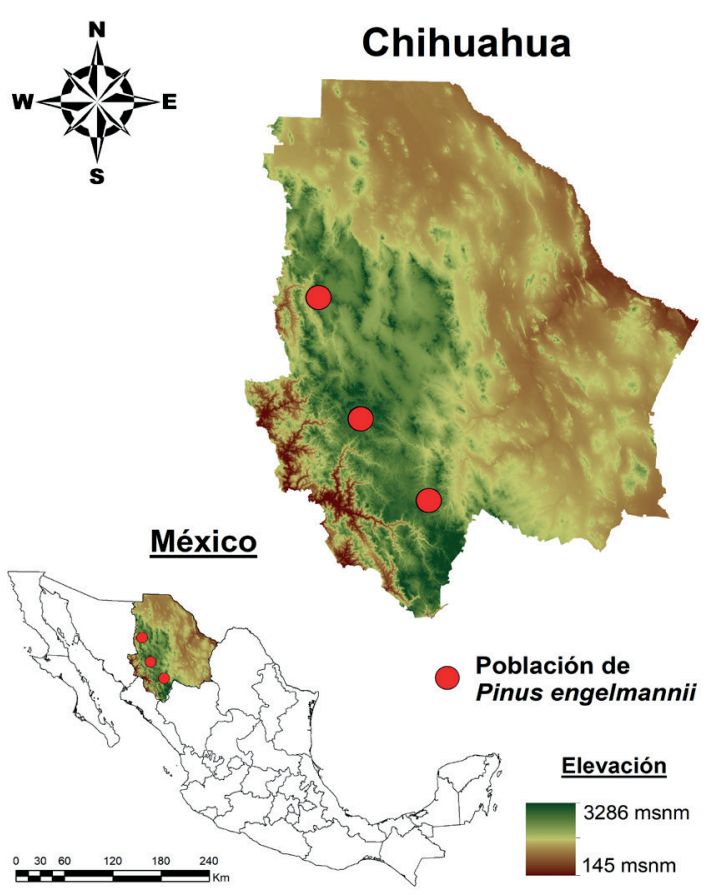

Figura 1. Localización geográfica de las poblaciones estudiadas de Pinus engelmannii en el estado de Chihuahua, México. 
NanoDrop 2000 (Thermo Fisher Scientific ${ }^{\mathrm{TM}}$, Wilmington, Delaware, EUA) y se ajustó a una concentración de 50 ng $\mu \mathrm{L}^{-1}$ con agua desionizada.

El análisis AFLP se realizó utilizando el Kit IRDye ${ }^{\circledR}$ Fluorescent AFLP ${ }^{\circledR}$ Kit for Large Plant Genome Analysis (LI-COR Bioscences). El análisis comenzó con la digestión de $2 \mu \mathrm{L}$ de ADN diluido por medio de las enzimas de restricción EcoRI y Msel; posteriormente, los fragmentos de ADN digeridos fueron ligados con adaptadores para EcoRI y Msel; después, se adhirió un nucleótido extra a los inicidores $(E c o R I+A$ y Msel $+\mathrm{C})$ para realizar la preamplificación. La amplificación selectiva se llevó a cabo mediante cuatro combinaciones de iniciadores marcados con fluorescencia: Msel + CTC - EcoRI + ACA, Msel + CAG - EcoRI + ACT, Msel + CTA - ECoRI + AGA, Msel + CTG EcoRI + AGC. La reacción en cadena de la polimerasa se realizó en un termociclador Cycler (Veriti, Applied Biosystems $^{\text {TM }}$ 2720, Foster City, California, EUA), con el siguiente programa: un ciclo de $94^{\circ} \mathrm{C}$ por $30 \mathrm{~s}, 65^{\circ} \mathrm{C}$ por $30 \mathrm{~s}, 72{ }^{\circ} \mathrm{C}$ por $1 \mathrm{~min} ; 12$ ciclos de $94^{\circ} \mathrm{C}$ por $30 \mathrm{~s}, 65^{\circ} \mathrm{C}$ por $30 \mathrm{~s}, 72^{\circ} \mathrm{C}$ por $1 \mathrm{~min} ; 23$ ciclos de $94^{\circ} \mathrm{C}$ por $30 \mathrm{~s}, 56^{\circ} \mathrm{C}$ por $30 \mathrm{~s}, 72{ }^{\circ} \mathrm{C}$ por $1 \mathrm{~min}$. Los productos de la amplificación selectiva $(2 \mu \mathrm{L})$ se mezclaron con $8 \mu \mathrm{L}$ de formamida y 1 $\mu \mathrm{L}$ de marcador Eco 700 GeneScan (Applied Biosystems). Finalmente, la separación y detección de fragmentos se realizó por medio de un secuenciador Genetic Analyzer 3730xl (Applied Biosystems).

\section{Análisis de la información}

Los electroferogramas obtenidos se organizaron en una matriz binaria de presencia (1) o ausencia (0) de bandas para realizar el análisis con el software Geneious versión R10. Se utilizó el sofware GenAlEx versión 6 (Peakall y Smouse, 2006) para obtener los estadísticos de diversidad, porcentaje de loci polimórficos, promedio de alelos por locus, número de alelos efectivos, índice de información de Shannon (I) y heterocigosidad esperada $\left(H_{\mathrm{e}}\right)$. Estos índices fueron estimados bajo el supuesto de que cada locus representa un par de alelos, cuando en una banda se encuentra presencia y ausencia de fragmento AFLP. La heterocigosidad esperada se estimó mediante el enfoque de Lynch y Milligan (1994), quienes utilizaron el índice de diversidad de Nei (1978) como el valor de heterocigosidad esperada. Los estadísticos de diversidad para cada población se compararon mediante la prueba Wilcoxon con corrección Bonferroni $(a=0.05)$.

La estructura genética de las poblaciones se infirió con base en el algoritmo de agrupamiento con un modelo Bayesiano mediante el software STRUCTURE versión 2.3.4 (Falush et al., 2007; Pritchard et al., 2000). El programa STRUCTURE se ejecutó 30 veces para cada k número de conglomerados genéticos y se analizó desde $k=1$ hasta $k=10$. En cada corrida se efectuaron 10,000 repeticiones de cadena de Markov-Monte Carlo (CMMC) y 20,000 periodos de rodaje (burn-in periods). Este análisis se llevó a cabo por medio de un modelo de mezcla y frecuencia de alelos correlacionados. Se consideró como número óptimo de $k$ grupos al que obtuvo el valor mayor de la probabilidad posterior promedio (log likelihood) y de $\Delta K_{\text {, }}$ de acuerdo con los criterios propuestos por Evanno et al (2005). Los valores de la probabilidad posterior promedio y $\Delta K$ se obtuvieron a través del sitio web Structure Harvester (Earl y vonHoldt, 2012). Se realizó un análisis de varianza molecular (AMOVA) (Excoffier et al., 1992), donde se comparó entre poblaciones y entre los grupos conformados en el análisis de estructura genética. Este análisis se llevó a cabo empleando el software Info-Gen (Balzarini y Di Rienzo, 2004). El índice de flujo genético se estimó con la fórmula $N_{m}=\left[0.25\left(1-\Phi_{S T}\right) /\left(\Phi_{S T}\right)\right]$ (Whitlock y Mccauley, 1999) utilizando los estadísticos $F\left(\Phi_{\text {ST }}\right)$ obtenidos del AMOVA.

Para detector posibles barreras eco geográficas que afecten el flujo genético entre poblaciones, los datos fueron analizados mediante el algoritmo de Monmonier, con ayuda de software Barrier versión 2.2 (Manni et al., 2004). Los valores de Bootstrap de cada barrera fueron calculados con 100 matrices de distancias genéticas Dice. Todos los análisis fueron realizados para cada especie por separado.

Cuadro 1. Ubicación geográfica y características ambientales de los lugares de procedencia de las tres poblaciones estudiadas de Pinus engelmannii en el estado de Chihuahua, México.

\begin{tabular}{llllllll}
\hline Ubicación & Latitud N & Longitud O & Población & Municipio & $\begin{array}{l}\text { Altitud } \\
(\mathrm{msnm})\end{array}$ & PPT $(\mathrm{mm})$ & TMA $\left({ }^{\circ} \mathrm{C}\right)$ \\
\hline Norte & 29.8179 & 108.2660 & Ejido El Largo & Madera & 2324 & 625 & 12.3 \\
Centro & 28.029 & 107.5999 & Mesa de Baboreachi & Bocoyna & 2229 & 724 & 12.2 \\
Sur & 26.9319 & 106.7202 & Carretera Balleza-Guachochi & Balleza & 1559 & 490 & 18.2 \\
\hline
\end{tabular}

PPT: precipitación media anual, TMA: temperatura media anual. Fuente: Climate-data.org (2019). 


\section{RESULTADOS Y DISCUSIÓN}

\section{Diversidad genética de las poblaciones}

El análisis AFLP produjo 1341 bandas en total para las dos combinaciones de iniciadores, de las cuales el $76 \%$ fueron polimórficas. Adicionalmente, se detectó un alto porcentaje de loci polimórficos dentro de las poblaciones (de 74 a $77 \%$ ). Estos resultados son mayores a los registrados con los mismos marcadores en otras especies de pinos; por ejemplo, Xu et al. (2008) encontraron 65 y $46 \%$ de loci polimórficos en P. echinata Mill y P. taeda L., respectivamente; así mismo, Rawat et al. (2014) encontraron 36 \% de loci polimórficos en P. roxburghi Sarg.

El valor de I total obtenido en este estudio es similar al encontrado para otras especies de pinos; Di y Wang (2013) evaluaron la diversidad de 20 poblaciones de P. tabulaeformis Carr. mediante marcadores AFLP y encontraron un I de 0.35; de igual manera, Androsiuk et al. (2015) encontraron un valor de I de 0.35 para 20 poblaciones de $P$. sylvestris $L$. distribuidas en 12 países europeos. Lo anterior sugiere que el nivel de diversidad genética encontrado en este estudio $(I=0.38)$ podría ser considerado como alto (Cuadro 2).

Los niveles obtenidos de heterocigosidad pueden considerarse como intermedios si se comparan con los encontrados en investigaciones previas con AFLP. Xu et al. (2008) encontraron valores de heterocigosidad esperada desde 0.08 hasta 0.17 en P. echinata Mill y P. taeda; en contraste, Kim et al. (2011) obtuvieron 0.35 de heterocigosidad esperada en P. monticola Dougl. Si se toma como base este intervalo, se podría sugerir que $P$. engelmannii presenta heterocigosidad moderada en el estado de Chihuahua $\left(H_{e}=0.25\right)$. Los resultados obtenidos en los parámetros de diversidad indicaron que las poblaciones del norte y sur de P. engelmannii poseen mayor nivel de diversidad que la población del centro, ya que obtuvieron el mayor porcentaje de loci polimórficos $(\mathrm{P} \leq 0.05)$, número de alelos por locus, índice de información de Shannon y heterocigosidad esperada (Cuadro 2).

\section{Estructura y diferenciación genética de las poblaciones}

El análisis de estructura genética (STRUCTURE) sugiere $k=2$ como el número apropiado de grupos, ya que obtuvo la mayor probabilidad posterior promedio (-16134.4) y el valor de $\Delta \mathrm{K}$ más alto (31.7). El grupo I quedó conformado por casi todos los árboles de las poblaciones del norte y centro, con excepción de tres individuos. Todos los árboles de la población sur quedaron integrados en el grupo II (Figura 2A); no obstante, los árboles de las tres poblaciones presentaron coeficientes de identidad para ambos grupos, lo cual es un indicador de que existe cierto grado de flujo genético en toda la sierra de Chihuahua. Lo anterior se puede observar en la gráfica de barras de la Figura 2A, donde los colores representan la proporción de la probabilidad de pertenecer a cada grupo. Por otro lado, el análisis de barreras genéticas (BARRIER) reveló la existencia de una discontinuidad genética (Bootstrap $=78$ $\%$ entre las poblaciones de $P$. engelmannii estudiadas. Esta barrera divide a la población del sur de las poblaciones del centro y norte (Figura 2B).

Los sistemas montañosos, ríos y barrancas ubicados entre los municipios de Guachochi y Guadalupe y Calvo podrían actuar como barrera geográfica con influencia en el intercambio genético entre la población del sur y las poblaciones del centro y norte. Con datos del modelo digital de elevación escala 1:50000 se estimó que la anchura de los cañones va de 4 a $60 \mathrm{~km}$, lo cual supone una distancia entre poblaciones de $P$. engelmannii de al menos $4 \mathrm{~km}$ en su porción más angosta y de 60 km en su porción más ancha (INEGI, 2019). Por otro lado, la distancia en línea recta entre las poblaciones muestreadas del norte y el centro es de 208 $\mathrm{km}$, mientras que entre la del centro y del sur es de $200 \mathrm{~km}$ (INEGI, 2019). Lo anterior concuerda con investigaciones previas, como la realizada por Yang et al. (2015), quienes

Cuadro 2. Parámetros de diversidad genética en tres poblaciones de Pinus engelmannii Carr. del estado de Chihuahua, México.

\begin{tabular}{lccccc}
\hline Población & $\begin{array}{c}\text { Porcentaje de loci } \\
\text { polimórficos }\end{array}$ & $\begin{array}{c}\text { Promedio de alelos } \\
\text { por locus }\end{array}$ & $\begin{array}{c}\text { Número de alelos } \\
\text { efectivos }\end{array}$ & I & $H_{e}$ \\
\hline Norte & 77 & $1.539 \mathrm{~b}$ & $1.41 \mathrm{a}$ & $0.382 \mathrm{a}$ & $0.249 \mathrm{a}$ \\
Centro & 74 & $1.494 \mathrm{c}$ & $1.38 \mathrm{~b}$ & $0.364 \mathrm{~b}$ & $0.237 \mathrm{~b}$ \\
Sur & 77 & $1.550 \mathrm{a}$ & $1.43 \mathrm{a}$ & $0.395 \mathrm{a}$ & $0.260 \mathrm{a}$ \\
Total & 76 & 1.528 & 1.41 & 0.380 & 0.249 \\
\hline
\end{tabular}

Valores en las columnas seguidos de la misma letra no son estadísticamente diferentes (Wilcoxon con corrección Bonferroni, $\mathrm{P} \leq \mathrm{0.05)}$ ) I: índice de información de Shannon, $H_{e}$ : heterocigosidad esperada o diversidad génica de Nei (1978). 
encontraron que barreras geográficas como sistemas montañosos y ríos han actuado como cuellos de botella en el intercambio genético entre poblaciones de $P$. henryi Mast y P. tabuliformis Carr. Así mismo, Wang et al. (2013) señalaron que factores geográficos y climáticos han sido causa importante de la diferenciación genética entre poblaciones de $P$. yunnanensis Franch.

El AMOVA determinó que existen diferencias significativas $(P \leq 0.05)$ entre la población del sur y las poblaciones del norte y centro; sin embargo, las poblaciones del norte y centro no fueron diferentes $(P=$ 0.28 ; Cuadro 3). Este resultado ratifica la sugerencia de que los programas de reforestación en el sur del estado deben realizarse con material genético de esa región, mientras que la reforestación en el norte y centro pueden realizarse con materiales de ambas regiones. Estos resultados concuerdan con los obtenidos por Ortiz-Olivas et al. (2017), quienes estudiaron la diversidad en rodales semilleros de $P$. engelmannii en el estado de Durango y encontraron una estructura genética espacial definida entre los rodales, por lo que recomendaron utilizar semilla local en los programas de reforestación de P. engelmannii. De igual manera, los resultados de este estudio coincidieron con lo señalado por Friedrich et al. (2018) para P. arizonica, quienes encontraron diferencias genéticas entre poblaciones del estado de Durango y recomendaron utilizar genotipos nativos para evitar la pérdida de las estructuras genéticas locales. El utilizar genotipos locales significa utilizar materiales adaptados e incrementar la probabilidad de éxito en programas de reforestación (Mijnsbrugge et al., 2010).

La variación entre poblaciones explicó $3.67 \%$ de la variación total, mientras que la variación dentro de las poblaciones explicó 96.33 \% (Cuadro 3). Este resultado indica que existe potencial para realizar programas de selección para obtener individuos con características sobresalientes. En pinos es común que la mayor parte de la variación total se encuentre dentro de poblaciones y

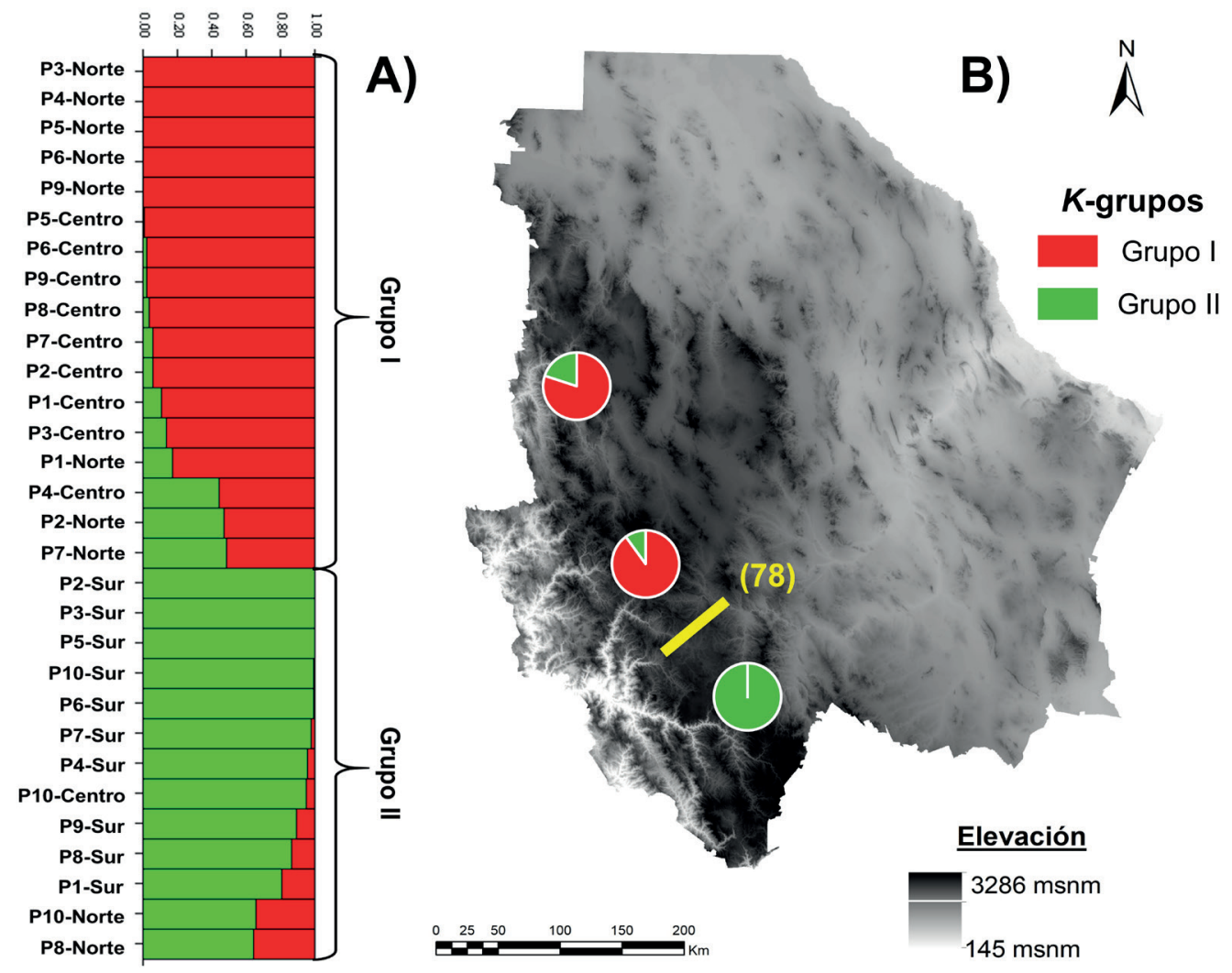

Figura 2. Estructura genética dentro y entre tres poblaciones de Pinus engelmannii en el estado de Chihuahua, México. A) Análisis STRUCTURE con $k=2$ realizado con base en 1341 fragmentos AFLP. Cada color representa la proporción de la probabilidad de pertenecer a cada grupo. B) Estructura en contexto geográfico, el área de las gráficas circulares representa el porcentaje de árboles de cada grupo. La barra amarilla representa una barrera en el flujo genético y el número es el valor de Bootstrap (1000 remuestreos) identificado mediante análisis BARRIER. El mapa fue generado con el modelo digital de elevación esc: 1:50000, con un tamaño de celda de 30 × 30 m (INEGI, 2019). 
la variación entre poblaciones represente solamente una pequeña proporción; un ejemplo de esto es lo reportado en P. oocarpa Schiede ex Schltdl., donde se encontró que la variación entre poblaciones solamente explicó 3.6 y $0.95 \%$ de la variación global con marcadores RAPD y AFLP, respectivamente (Díaz et al., 2001). Otro ejemplo es mencionado por Androsiuk et al. (2015), quienes encontraron que la variación entre poblaciones de $P$. sylvestris representó $5.3 \%$ de la variación total.

El índice de flujo genético $\left(\mathrm{N}_{\mathrm{m}}\right)$ promedio entre las tres poblaciones fue de 6.56 (Cuadro 3); sin embargo, al comparar entre poblaciones se encontró un flujo genético alto entre las poblaciones del norte y centro (40.7 individuos por generación), en contraste con el flujo genético que comparten estas dos poblaciones con la población del sur (3.38 y 5.74). Estos resultados son consistentes con el patrón de agrupamiento obtenido en el análisis STRUCTURE. De igual manera, los índices de flujo genético concuerdan con los resultados del análisis BARRIER, el cual reveló una barrera geográfica al flujo genético que separa a la población del sur de las poblaciones del centro y norte; a pesar de ello, el flujo genético entre poblaciones encontrado en este estudio se puede considerar alto si se compara con los valores que se han encontrado en otras coníferas; por ejemplo, Di y Wang (2013) encontraron un flujo genético de 0.57 individuos por generación entre poblaciones de P. tabulaeformis. En este mismo sentido, Xue et al. (2005) obtuvieron un índice de flujo de genético de 0.96 individuos por generación en poblaciones de Picea asperata Mast.

La diversidad y el flujo genético encontrados en este estudio podrían deberse a que los pinos se caracterizan por poseer un mecanismo eficiente de reproducción mediante la dispersión de polen a través del viento (Parker et al., 2001). Investigaciones previas han reportado que el polen de los pinos puede dispersarse a grandes distancias de la fuente de origen y ser aún viable. Williams (2010) encontró que $P$. taeda puede dispersar polen hasta $41 \mathrm{~km}$ de la fuente, Robledo-Arnuncio (2011) señaló que el polen de $P$. sylvestris puede viajar hasta $100 \mathrm{~km}$ de su población de origen y Varis et al. (2009) detectaron granos de polen viables a más de $600 \mathrm{~km}$ de la fuente en P. sylvestris. Por otro lado, pájaros y mamíferos pueden dispersar las semillas de pinos, lo cual también provoca flujo genético y aumenta la diversidad (McCune, 1988). Existen además datos de que los pájaros cascanueces (Nucifraga sp.) pueden transportar semillas de pinos a más de $22 \mathrm{~km}$ (Tomback y Linhart, 1990).

\section{CONCLUSIONES}

Existen diferencias de diversidad genética entre las poblaciones del sur y del norte con respecto a la población del centro del estado de Chihuahua. La amplia diversidad genética encontrada dentro de poblaciones muestra que existe potencial para realizar programas de selección de

Cuadro 3. Análisis molecular de varianza (AMOVA) entre tres poblaciones de Pinus engelmannii y entre grupos formados por análisis STRUCTURE, calculado con 1341 fragmentos AFLP.

\begin{tabular}{|c|c|c|c|c|c|c|c|}
\hline Fuente de variación & $\mathrm{GL}$ & SC & CV & PV & $\mathrm{P}$ & $\Phi_{\text {ST }}$ & $\mathrm{N}_{\mathrm{m}}$ \\
\hline \multicolumn{8}{|l|}{ AMOVA general } \\
\hline Entre poblaciones & 2 & 776182.67 & 10701.43 & 3.67 & 0.01 & \multirow{2}{*}{0.04} & \multirow{2}{*}{6.56} \\
\hline Dentro de poblaciones & 27 & 7589079.2 & 281077.01 & 96.33 & 0.01 & & \\
\hline \multicolumn{8}{|l|}{ AMOVA norte vs centro } \\
\hline Entre poblaciones & 1 & 286539.2 & 1648.96 & 0.61 & 0.28 & \multirow{2}{*}{0.01} & \multirow{2}{*}{40.7} \\
\hline Dentro de poblaciones & 18 & 4860893.6 & 270049.64 & 99.39 & 0.001 & & \\
\hline \multicolumn{8}{|l|}{ AMOVA norte vs sur } \\
\hline Entre poblaciones & 1 & 455909 & 17881.74 & 6.06 & 0.0011 & \multirow{2}{*}{0.06} & \multirow{2}{*}{3.38} \\
\hline Dentro de poblaciones & 18 & 4987648.4 & 277091.58 & 93.94 & 0.0005 & & \\
\hline \multicolumn{8}{|l|}{ AMOVA centro vs sur } \\
\hline Entre poblaciones & 1 & 421789.8 & 12557.65 & 4.07 & 0.045 & \multirow{2}{*}{0.04} & \multirow{2}{*}{5.74} \\
\hline Dentro de poblaciones & 16 & 5331839.2 & 296213.29 & 95.93 & 0.042 & & \\
\hline \multicolumn{8}{|c|}{ AMOVA grupos estructura $\mathrm{k}=2$} \\
\hline Entre grupos & 1 & 678944.31 & 25322.55 & 8.17 & 0.0028 & \multirow{2}{*}{0.08} & \multirow{2}{*}{2.80} \\
\hline Dentro de grupos & 27 & 7688429.82 & 284756.66 & 91.83 & 0.0025 & & \\
\hline
\end{tabular}


genotipos sobresalientes para características de interés. La población del sur se diferencia genéticamente de las poblaciones del norte y centro, las cuales presentan estructuras genéticas similares; en este sentido, los programas de reforestación en el sur del estado deben llevarse a cabo con materiales locales, mientras que la reforestación en el norte y centro pueden realizarse con materiales de ambas regiones. El alto intercambio genético que existe entre poblaciones de $P$. engelmannii puede ser una de las causas de la diversidad de esta especie en la región de estudio. Por otro lado, la disminución en el flujo genético entre la población del sur con las poblaciones del centro y norte podría estar asociada con la presencia de barreras orográficas como las barrancas y cañones de Chihuahua.

\section{AGRADECIMIENTOS}

Los autores agradecen el apoyo de la Dra. June Simpson y el equipo de trabajo del laboratorio de Genética Molecular del Centro de Investigación y de Estudios Avanzados del Instituto Politécnico Nacional (CINVESTAV), Unidad Irapuato.

\section{BIBLIOGRAFÍA}

Androsiuk P., S. Ciągło-Androsiuk and L. Urbaniak (2015) Genetic diversity and differentiation of Pinus sylvestris L. from the IUFRO 1982 provenance trial revealed by AFLP analysis. Archives of Biological Sciences 67:1237-1249, https://doi.org/10.2298/ ABS150319100A

Ávila-Flores I. J., J. C. Hernández-Díaz, M. S. González-Elizondo, J. A. Prieto-Ruíz and C. Wehenkel (2016) Pinus engelmannii Carr. in Northwestern México: a review. Pakistan Journal of Botany 48:2159-2166

Balzarini M. y J. Di Rienzo (2004) Info-Gen: Software para análisis estadístico de datos genéticos. Universidad Nacional de Córdoba. Córdoba, Argentina. http://www.info-gen.com.ar (Mayo 2019).

Bilgen B. B. and N. Kaya (2017) Genetic diversity among Pinus sylvestris L. populations and its implications for genetic conservation: comparison of nuclear and chloroplast microsatellite markers Fresenius Environmental Bulletin 26:6873-6881.

Climate-data.org (2019) Clima Chihuahua: Largo Maderal, San Juanito, Balleza. http://es.climate-data.org/america-del-norte/mexico/ chihuahua-10/ (Mayo 2019).

CONAFOR, Comisión Nacional Forestal (2009) Inventario Nacional Forestal y de Suelos de México 2004-2009. Comisión Nacional Forestal Zapopan, Jalisco, México. 206 p.

Di X. Y. and M. B. Wang (2013) Genetic diversity and structure of natural Pinus tabulaeformis populations in North China using amplified fragment length polymorphism (AFLP). Biochemical Systematics and Ecology 51:269-275, https://doi.org/10.1016/j. bse.2013.09.013

Díaz V., L. M. Muñiz and E. Ferrer (2001) Random amplified polymorphic DNA and amplified fragment length polymorphism assessment of genetic variation in Nicaraguan populations of Pinus oocarpa. Molecular Ecology 10:2593-2603, https://doi.org/10.1046/ j.0962-1083.2001.01390.x

Earl D. A. and B. M. vonHoldt (2012) STRUCTURE HARVESTER: a website and program for visualizing STRUCTURE output and implementing the Evanno method. Conservation Genetics Resources 4:359-361, https://doi.org/10.1007/s12686-0119548-7
Evanno G., S. Regnaut and J. Goudet (2005) Detecting the number of clusters of individuals using the software STRUCTURE: a simulation study. Molecular Ecology 14:2611-2620, https://doi. org/10.1111/j.1365-294X.2005.02553.x

Excoffier L., P. E. Smouse and J. M. Quattro (1992) Analysis of molecular variance inferred from metric distances among DNA haplotypes: application to human mitochondrial DNA restriction data. Genetics 131:479-491

Falush D., M. Stephens and J. K. Pritchard (2007) Inference of population structure using multilocus genotype data: dominant markers and null alleles. Molecular Ecology Notes 7:574-578, https://doi. org/10.1111/j.1471-8286.2007.01758.x

FAO, Organización de las Naciones Unidas para la Agricultura y la Alimentación; FLD, Bosque y Paisaje de Dinamarca y Bioversity International (2007) Conservación y Manejo de los Recursos Genéticos Forestales. Vol. 1: Visión General, Conceptos y Algunos Métodos Sistemáticos. Instituto Internacional de Recursos Fitogenéticos. Roma, Italia. $111 \mathrm{p}$

Friedrich S. C., J. C. Hernández-Díaz, L. Leinemann, J. A. Prieto-Ruíz and C. Wehenkel (2018) Spatial genetic structure in seed stands of Pinus arizonica Engelm. and Pinus cooperi Blanco in the State of Durango, Mexico. Forest Science 64:191-202, https://doi. org/10.1093/forsci/fxx007

Hernández-Velasco J., J. C. Hernández-Díaz, M. Fladung, Á. Cañadas-López, J. Á. Prieto-Ruíz and C. Wehenkel (2017) Spatial genetic structure in four Pinus species in the Sierra Madre Occidental, Durango, México. Canadian Journal of Forest Research 47:73-80, https:// doi.org/10.1139/cjfr-2016-0154

INEGI, Instituto Nacional de Estadística y Geografía (2019) Continuo de elevaciones mexicano (CEM). Instituto Nacional de Estadística y Geografía. Aguascalientes, Aguascalientes. https://www.inegi. org.mx/app/geo2/elevacionesmex/ (Noviembre 2019).

Ivetić V., J. Devetaković, M. Nonić, D. Stanković and M. Šijačić-Nikolić (2016) Genetic diversity and forest reproductive material - from seed source selection to planting. iForest-Biogeosciences and Forestry 9:801-812, https://doi.org/10.3832/ifor1577-009

Kim M. S., B. A. Richardson, G. I. McDonald and N. B. Klopfenstein (2011) Genetic diversity and structure of western white pine (Pinus monticola) in North America: a baseline study for conservation, restoration, and addressing impacts of climate change. Tree Genetics and Genomes 7:11-21, https://doi.org/10.1007/ s11295-010-0311-0

Lynch M. and B. G. Milligan (1994) Analysis of population genetic structure with RAPD markers. Molecular Ecology 3:91-99, https://doi. org/10.1111/j.1365-294X.1994.tb00109.x

Manni F., E. Guérard and E. Heyer (2004) Geographic patterns of (genetic, morphologic, linguistic) variation: how barriers can be detected by using Monmonier's algorithm. Human Biology 76:173-190, https://doi.org/10.1353/hub.2004.0034

McCune B. (1988) Ecological diversity in North American pines. American Journal of Botany 75:353-368, https://doi. org/10.1002/j.1537-2197.1988.tb13450.x

Méndez-Neri M., C. Ramírez-Herrera, J. J. Vargas-Hernández, T. MartínezTrinidad, J. López-Upton y P. A. López (2020) Diversidad genética en dos huertos semilleros de Pinus patula Schiede ex Schltdl. et Cham. Revista Fitotecnia Mexicana 43:113-119, https://doi. org/10.35196/rfm.2020.1.113-119

Mijnsbrugge V. K., A. Bischoff and B. Smith (2010) A question of origin: where and how to collect seed for ecological restoration. Basic and Applied Ecology 11:300-311, https://doi.org/10.1016/j. baae.2009.09.002

Nei M. (1978) Estimation of average heterozygosity and genetic distance from a small number of individuals. Genetics 89:583590.

Ortiz-Olivas M. E., J. C. Hernández-Díaz, M. Fladung, Á. Cañadas-López, J. Á. Prieto-Ruíz and C. Wehenkel (2017) Spatial genetic structure within and among seed stands of Pinus engelmannii Carr. and Pinus leiophylla Schiede ex Schltdl. \& Cham, in Durango, Mexico. Forests 8:22, https://doi.org/10.3390/f8010022

Parker K. C., J. L. Hamrick, A. J. Parker and J. D. Nason (2001) Fine scale genetic structure in Pinus clausa (Pinaceae) populations: effects of disturbance history. Heredity 87:99-113, https://doi. org/10.1046/j.1365-2540.2001.00914.x 
Pazouki L., P. S. Shanjani, P. D. Fields, K. Martins, M. Suhhorutšenko, H. Viinalass and Ü. Niinemets (2016) Large within-population genetic diversity of the widespread conifer Pinus sylvestris at its soil fertility limit characterized by nuclear and chloroplast microsatellite markers. European Journal of Forest Research 135:161-177, https://doi.org/10.1007/s10342-015-0928-5

Peakall R. and P. E. Smouse (2006) GENALEX 6: genetic analysis in excel. Population genetic software for teaching and research. Molecular Ecology Notes 6:288-295, https://doi.org/10.1111/ j.1471-8286.2005.01155.x

Prieto R. J. A., E. H. Cornejo O., P. A. Domínguez C., J. J. Návar C., J. G. Marmolejo M. y J. Jiménez P. (2004) Estrés hídrico en Pinus engelmannii Carr., producido en vivero. Investigación Agraria. Sistemas y Recursos Forestales 13:443-451.

Pritchard J. K., M. Stephens and P. Donnelly (2000) Inference of population structure using multilocus genotype data. Genetics 155:945959.

Quiñones-Pérez C. Z., C. Sáenz-Romero and C. Wehenkel (2014) Influence of neighbouring tree species on AFLP variants of endangered Picea chihuahuana Martínez populations on the Sierra Madre Occidental, North-Western Mexico. Polish Journal of Ecology 62:55-65, https://doi.org/10.3161/104.062.0106

Rawat A., S. Barthwal and H. S. Ginwal (2014) Comparative assessment of SSR, ISSR and AFLP markers for characterization of selected genotypes of Himalayan Chir pine (Pinus roxburghii Sarg.) based on resin yield. Silvae Genetica 63:94-108, https://doi. org/10.1515/sg-2014-0013

Robledo-Arnuncio J. J. (2011) Wind pollination over mesoscale distances: an investigation with Scots pine. New Phytologist 190:222-233, https://doi.org/10.1111/j.1469-8137.2010.03588.x

Rodríguez-Peña R. A., R. L. Johnson, L. A. Johnson, C. D. Anderson, N. J. Ricks, K. M. Farley, ... and M. R. Stevens (2018) Investigating the genetic diversity and differentiation patterns in the Penstemon scariosus species complex under different sample sizes using AFLPs and SSRs. Conservation Genetics 19:1335-1348, https://doi.org/10.1007/s10592-018-1103-6

Sánchez-González A. (2008) Una visión actual de la diversidad y distribución de los pinos de México. Madera y Bosques 14:107-
120, https://doi.org/10.21829/myb.2008.1411222

St.Clair J. B. and G. T. Howe (2011) Strategies for conserving forest genetic resources in the face of climate change. Turkish Journal of Botany 35:403-409, https://doi.org/10.3906/bot-1012-98

Tomback D. F. and Y. B. Linhart (1990) The evolution of bird-dispersed pines. Evolutionary Ecology 4:185-219, https://doi.org/10.1007/ BF02214330

Vangestel C., A. Vázquez-Lobo, P. J. Martínez-García, I. Calic, J. L. Wegrzyn and D. B. Neale (2016) Patterns of neutral and adaptive genetic diversity across the natural range of sugar pine (Pinus lambertiana Dougl.). Tree Genetics and Genomes 12:51, https:// doi.org/10.1007/s11295-016-0998-7

Varis S., A. Pakkanen, A. Galofré and P. Pulkkinen (2009) The extent of South-North pollen transfer in Finnish Scots pine. Silva Fennica 43:717-726, https://doi.org/10.14214/sf.168

Wang B., J. F. Mao, W. Zhao and X. R. Wang (2013) Impact of geography and climate on the genetic differentiation of the subtropical pine Pinus yunnanensis. PLOS ONE 8(6):e67345, https://doi. org/10.1371/journal.pone.0067345

Whitlock M. C. and D. E. McCauley (1999) Indirect measures of gene flow and migration: $F_{s T} \neq 1 /(4 N m+1)$. Heredity $82: 117-125$, https:// doi.org/10.1038/sj.hdy.6884960

Williams C. G. (2010) Long distance pine pollen still germinates after meso scale dispersal. American Journal of Botany 97:846-855, https://doi.org/10.3732/ajb.0900255

Xu S., C. G. Tauer and C. D. Nelson (2008) Genetic diversity within and among populations of shortleaf pine (Pinus echinata Mill.) and loblolly pine (Pinus taeda L.). Tree Genetics and Genomes 4:859-868, https://doi.org/10.1007/s11295-008-0158-9

Xue X., Y. Wang, H. Korpelainen and C. Li (2005) Assessment of AFLPbased genetic variation in the populations of Picea asperata. Silvae Genetica 54:24-30, https://doi.org/10.1515/sg-20050004

Yang L., Z. L. Liu, J. Li and R. J. Dyer (2015) Genetic structure of Pinus henryi and Pinus tabuliformis: natural landscapes as significant barriers to gene flow among populations. Biochemical Systematics and Ecology 61:124-132, https://doi.org/10.1016/j. bse.2015.06.003 